\title{
Visualization methods for sharing knowledge pieces and relationships based on biological models
}

\author{
Masao Usuki and Kozo Sugiyama \\ School of Knowledge Science, Japan Advanced Institute of Science and Technology \\ (JAIST) 1-1, Asahidai, Tatsunokuchi, Ishikawa, 923-1292, Japan \\ (Usuki, Sugi)@jaist.ac.jp
}

\begin{abstract}
We study visualization methods for sharing knowledge pieces and relationships. Our purpose is to grasp informational structures readily. We develop algorithms based on a flocking model which simulates birds or fishes behavior in making a group. We implement two visualization systems for aesthetically draw diagrams of weak and strong structures (i.e. clusters and graphs). We evaluate performance of the systems.
\end{abstract}

\section{Introduction}

Recently an organization tends to be constituted with multi-site offices. This means that it is increasingly needed to provide a context-sharing environment in a distributed community that is connected through networks where formal and informal knowledge is exchanged each other without delay. Specially informal knowledge such as e-mails, news, chats and so on is very important and an effective informal communication support tool is desired since informal communication is becoming scarce in a distributed community. Such the informal knowledge usually is piecewise, structured (weakly or strongly), and frequently updated. User interfaces of informal communication tools should attain to show structures such as clusters and relationships among piecewise knowledge aesthetically and dynamically. Moreover, they should attain both to make the user easily aware knowledge presented and to avoid disturbing the user in doing one's main work.

Methods for automatic visualization of structures so far have been developed for relatively simple structures such as trees, digraphs and undirected graphs basing on mechanical and physical models[1]. In this paper we propose two novel and automatic visualization methods to well represent pieces and relationships of knowledge basing upon biological models. One is for the case of weak structures (i.e. clusters) which simulate flocking of fishes. The other one is for the case of strong structures (i.e. more complicated graphs) which refer physiological models in a cell called 'signal transduction pathways'.

In the subsequent sections, we describe how to visualize clusters and complicated graphs and discuss the effectiveness of them for user interfaces of representing knowledge pieces and relationships. 


\section{Visualization method for clusters by simulating flocking of fishes}

In the automatic visualization study, there has been no method for automatically visualizing clusters where a flocking model is used. In this section we propose a new method to visualize clusters as schools of fishes based upon Reynolds[2]. When each mate judges a local situation and acts in a flock of fishes, a group is formed automatically. The basic flocking model proposed by Reynolds consists of three simple steering behaviors as follows, which describe how an individual mate maneuvers based on the positions and velocities of its nearby flockmates.

1. Separation: steer to avoid crowding local flockmates

2. Cohesion: steer to move toward the average position of local flockmates

3. Alignment: steer towards the average heading of local flockmates

Figs.1 (a), (b) and (c) illustrate the three school rules of separation, cohesion, and alignment, respectively. In these figures a circle shows an area perceived by a black fish. A vector incident to the black fish means the direction and distance of the black fish's movement. The vectors of each individual mate are calculated from the three simple steering behaviors and these vectors are integrated as an vector. Each mate is moved little by little according to an integrated vector. Fig. 2 (a), (b) and (c) shows a snapshot of 3D visualization, a snapshot of 3D stereo graphics visualization, and a stereo graphics equipment respectively.

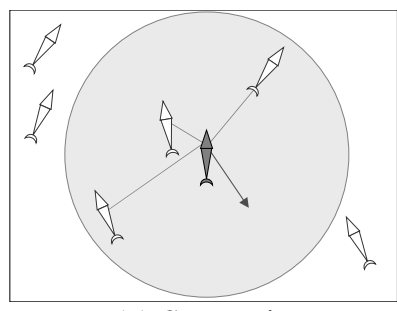

(a) Separation

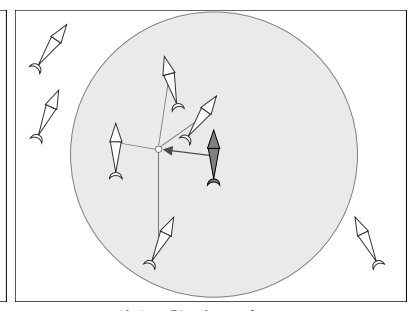

(b) Cohesion

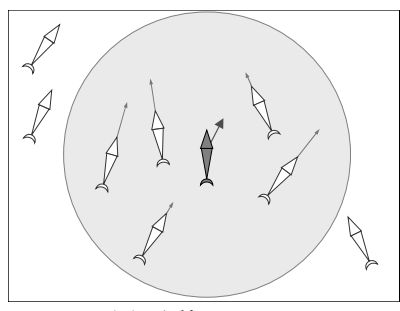

(c) Alignment

Fig. 1. Three school behaviors

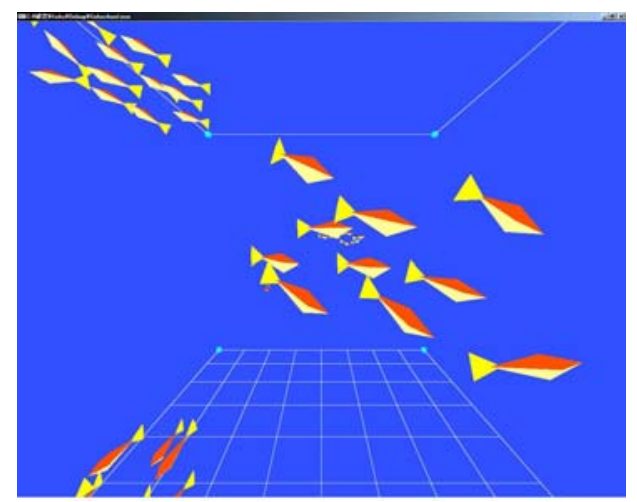

(a) 3D visualization 


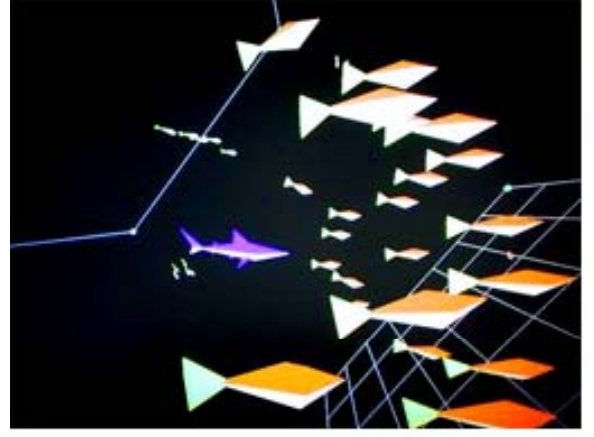

(b) 3D stereo graphics visualizaion

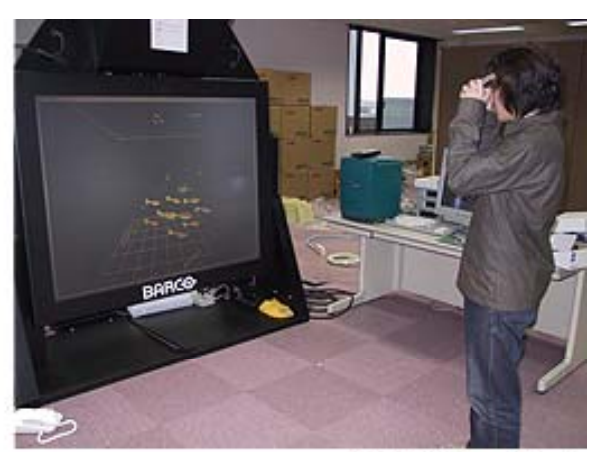

(c) 3D stereo graphics equipment

Fig. 2. Visualization of clustered pieces

The result of simulations of fish flocking shows that each kinds of fishes can be easily grouped in positions and movements of groups differ from each other. We can change parameters of the three flocking behaviors for each kind of fishes so that we can easily identify groups of fishes. This means that the method can represent clusters well. In terms of a user interface for an informal communication tool, the following usage of the method might be effective: each fish is linked to a knowledge piece and a kind of fishes is corresponded to a kind of messages or respective member. Sometimes a big fish like a shark traverses in a display with a specific behavior (see Fig.2(b)). We can assign an emergency news with a shark since it is easy for everybody to recognize it. Moreover, as seeing fish behaviors are comfortable for most people, we can expect relaxation effects that might avoid disturbance of one's main task. We need further research to evaluate this method from the above view points.

\section{Visualization method for complex structures by referring a biological model}

In the previous section we described a visualization method for weak structures (or clusters) by simulating flocking of fishes. In this section we discuss a visualization method for more complicated structures (or graphs). For this purpose we need real examples of diagrams of complicated structures since automatic visualization methods so far have been developed for rather simple graphs such as trees, digraphs, and undirected graphs. We made a survey and found various diagrams used in the biology that seemed to be useful for a user interface. Especially diagrams of signal transduction pathways attracted attentions. Signal transduction pathways consist of interactions between substances. They have an important function to obain information from external world and transmit information from neighbor to neighbor in a cell in order to cope with the external world. We considered that this situation of information relationships was similar to the situation of informational dynamics of 
each member of an organization. Therefore, we adopted this diagram as a target for our visualization. Fig. 3 shows various diagrams appeared in the literature $[3,4]$.

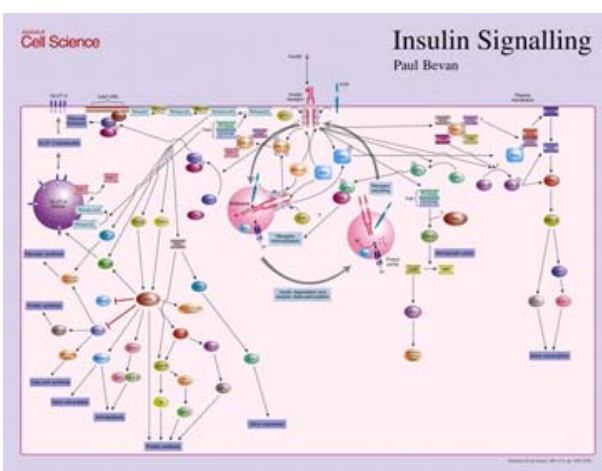

(a) Insulin signalling[3]



(b) T cell receptor signalling[4]

Fig. 3. Examples of diagrams in the biology

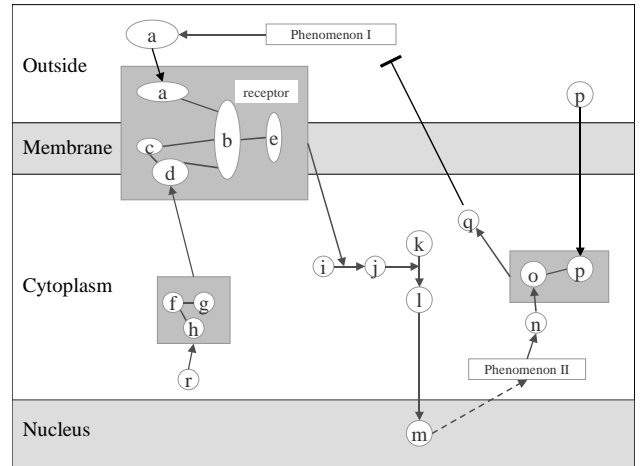

Fig. 4. Our model of a complicated structure: This diagram can represent clusters of nodes, directed and undirected relationships among nodes, layered layout of each element from outside to core, relationships between edges and so on. Therefore, this diagram model is so general and so it is effective to represent structures among knowledge pieces and relationships in an office or a member.

Currently diagrams of signal transduction pathways as shown Fig.3 are created by manual works. However, it is difficult for experts to create a diagram because there exist many elements consisting of signal transduction pathways. Therefore, any automatic method of creating a diagram is needed. Fig. 4 illustrates our model of a complicated graph that is devised referring real diagrams appeared in the literature. In the diagram figurations that abstract a cell are used frequently. A layered structure that represents a cell is also used in the diagram. Substances and the phenomena are expressed by ellipses on a layered structure. The complex mate with two or more substances is drawn as a rectangle which includes two or more ellipses. The relation between substances are expressed with line segments. An arrow expresses the direction of a relation. In the diagram, an ellipse and a rectangle can exist in the form 
over the boundary of layer structure. And a relation from an arrow to an arrow can exist. Thus the diagram is so complicated and more general when comparing with diagrams that are targeted by the existing drawing methods[1].

Aesthetic criteria for visualizing a diagram presented in Fig.4 are also complicated: avoid overlapping among nodes, avoid overlapping between nodes and edges, minimum separation between nodes, reduce the number of edge crossings, keep edge lengths uniformly, clarify flows around a node and so on. Fig. 5 illustrates a criterion: to clarify flows around a node.

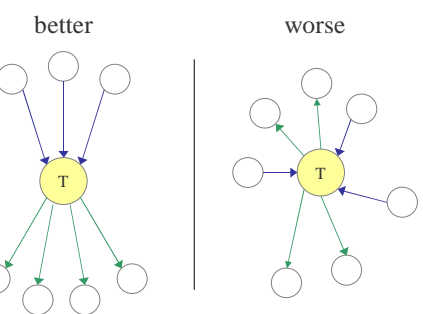

Fig. 5. Criterion for clarifying flows around a node

The flocking model described in the previous section is also applied to automatically visualize a diagram. Each mate is considered as an ellipse showing a substance. And a flock mate is considered as a connected ellipse. Each ellipse is moved little by little according to the sum of five calculated vectors: Separation(v), Cohesion(v), Alignment(v), Connection(v), and Rotation(v) for each ellipse $v$. The followings express formulae for three simple steering behaviors.

$$
\begin{gathered}
\text { Separation }(v)=\frac{1}{\left|V^{\prime}\right|} \sum_{x \in V^{\prime}} \frac{k-|\overrightarrow{x v}|}{|\overrightarrow{x v}|} \overrightarrow{x v} \\
V^{\prime}=\{x \in V|| \overrightarrow{x v} \mid \leq k\} \\
\text { Cohesion }(v)=\frac{1}{|C|} \sum_{x \in C} \overrightarrow{v x} \\
\text { Alignment }(v)=\frac{1}{|D|} \sum_{x \in D}\left\{\begin{array}{l}
\overrightarrow{v x}-\left|\frac{\overrightarrow{v x}}{\overrightarrow{A_{x}}}\right| \overrightarrow{A_{x}} \text { if } v \text { is source } \\
|\overrightarrow{v x}| \overrightarrow{A_{x}} \mid \overrightarrow{A_{x}} \text { if } v \text { is target }
\end{array}\right.
\end{gathered}
$$


Here $V$ is the set of all nodes. $C$ is a set of nodes that connected to node $v$. $D$ is a set of nodes that connected to node $v$ by directed edges. $\overrightarrow{A_{x}}$ is an average vector of directed edges that connected node $x$

Our model is complicated but our algorithm is simple. Its outline is as follows.

\section{algorithm: LAYOUT_LIKE_CELL}

Input: a compound graph $G=(V, E)$, layer-constraints for nodes $V$;

Output: Diagram $D$ of $G$;

1. configure layers considering the number of nodes in each layer and position nodes randomly;

2. repeat $n$ times;

2.1. calculate vectors Separation(v), Cohesion(v), Alignment(v), Connection(v), and Rotation(v), and obtain a sum of them for each node $v$;

2.2. move each node a little according to the sum vector;

3. draw a diagram adjusting the widths of layers and the sizes of cluster nodes.

We performed experiments to evaluate the performance of our automatic visualization method. Five real biological data in the literature (average node $=35.1$, average cluster $=4.0$, average edge $=39.8$ ) were used in the experiments. In spite of the complicatedness of the diagram proposed in this section, we had the following good performance:

1. The average success rate of placement of nodes to layers is $99.16 \%$.

2. The average success rate to avoid overlapping of nodes is $99.47 \%$.

3. The average success rate to avoid crossings among edges is $93.29 \%$.

The values of each layout criterion were considerably good. However, it could not attain perfectly. Therefore, a function for editing diagrams is needed. In Fig. 6 results automatically obtained by our method are shown.

\section{Concluding remarks}

We have proposed two novel methods for visualizing piecewise knowledge with weak and strong structures based on biological models. Biological models were good sources for considering new paradigms in visualization studies of structured information. We adopt clusters as weak structures and complex graphs that were found through the extensive survey of literatures. The complex graphs have powerful ability to represent considerably general structures. We have implemented both methods and made preliminary evaluations. We have confirmed that the flocking model of fishes is effective to visualize clusters of nodes and the combination of flocking model and other existing techniques for visualizing graphs can satisfy 
aesthetic criteria sufficiently. These methods developed in the study will be useful not only for a user interface of informal communication support tool but also for other extensive applications.

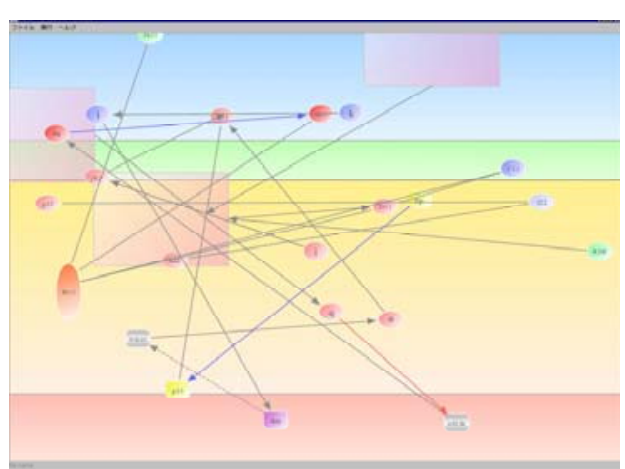

(a.1) initial state

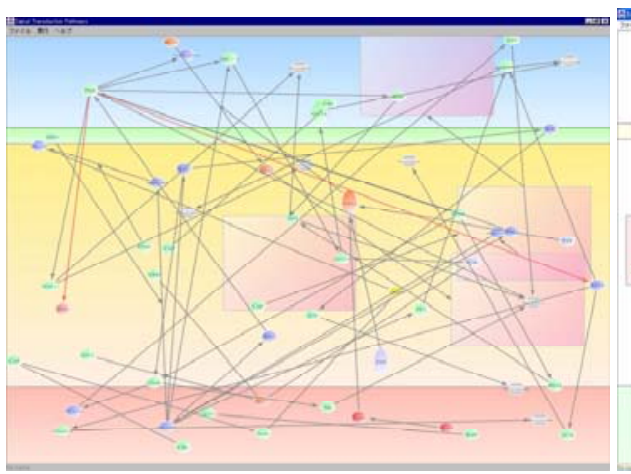

(b.1) initial state

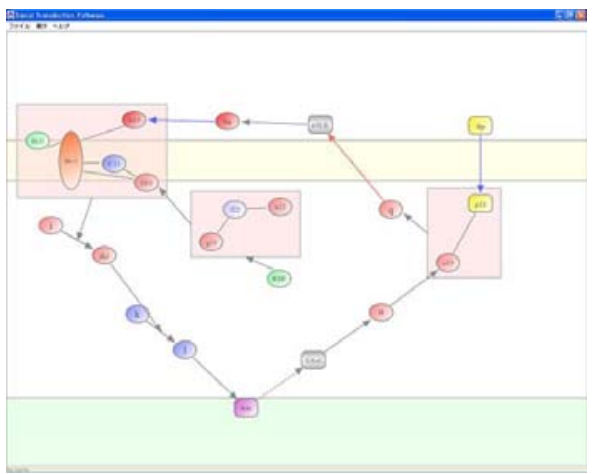

(a.2) final state

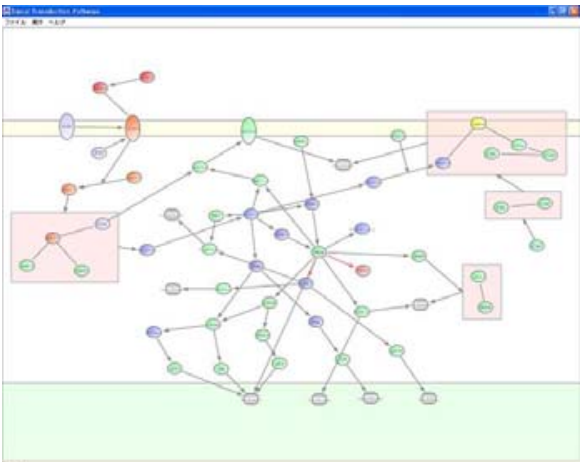

(b.2) final state

Fig. 6. Snapshots of visualization of complex diagrams

\section{References}

1. Sugiyama, K: Graph drawing and applications for software and knowledge engineers, World Scientific, 220p, 2002.

2. Reynolds, C. W.: Flocks herds, and schools: A distributed behavioral model, Computer Graphics, Vol.21, No.4, 21-35, 1987.

3. Bevan, P.: Insulin signalling, J. of Cell Science, No.114, 1601-1602, 2001.

4. Lin, J. and Weiss, A.: T cell receptor signalling, J. of Cell Science, No114, 243244, 2001. 